\title{
THE SOLAR IRRADIATION RECORD \\ IN LUNAR DUST GRAINS
}

\author{
J. BORG and B. VASSENT \\ Centre de Spectrométrie de Masse du C.N.R.S.-91 ORSAY-France
}

\begin{abstract}
Comparative studies of the distribution of latent and etched tracks in lunar grains from five different size fractions of three lunar fine samples and of six lunar dust samples taken at different depths in core tube 12028 have been performed by using transmission and scanning electron microscopies. Two very different sets of etching conditions were used: a slight etching was applied for transmission microscopy but a much stronger etching was used for scanning microscopy. We observed: (1) a definite stratigraphy in the core tube, both in the latent and etched track distributions; (2) striking differences between the densities of the latent and slightly etched tracks $\left(\geqslant 10^{10} \operatorname{tracks~}^{-2}\right)$ and those of the strongly etched tracks, ranging from $\simeq 10^{8}$ to $5.10^{9}$ tracks $\mathrm{cm}^{-2}$; (3) a lack of correlation between the grain size and the density of strongly etched tracks observed on the external surface of grains from sample 12032 ; (4) no variation of the density of the tracks with the depth inside a grain. Some implications of the present results concerning the ancient low energy solar cosmic rays and the fabric of the lunar soil will be briefly discussed.
\end{abstract}

\section{Introduction}

This paper describes our work on fossil nuclear particle tracks in lunar dust grains which is a part of the Orsay group investigations concerning the galactic and solar irradiation records in extraterrestrial matter.

Before our transmission electron microscope studies of meteoritic and lunar samples $[1,2,3,4,5]$, only etched tracks were studied [6] by scanning electron microscopy and optical microscopy in view of understanding the lunar regolith formation and determining some characteristics of the ancient solar flare cosmic rays.

In the present work, both latent and etched tracks have been studied in silicate grains extracted from five size fractions - 100, 200, 325, 400 and 400 Mesh residue - of the Apollo 11 and 12 lunar dust and core tube samples. Latent and very slightly etched tracks were observed directly by $100 \mathrm{keV}$ and $1 \mathrm{MeV}$ transmission electron microscopy in the finest feldspar and pyroxene grains. In the coarser grains the density of etched tracks was determined by high resolution scanning electron microscopy first on the external surface of the grains and then on internal surfaces obtained by polishing.

The main purposes of these studies were: (1) to discuss dynamic processes acting in the lunar regolith and more particularly to look for eventual differences in the irradiation history of the dust grains as a function either of their size or of their depth in core tube 12028 ; (2) to detect different types of solar radiations and more particularly to try to find evidence for the existence of low energy heavy ions, related to the suprathermal solar protons recently discovered by Franck [7] in the interplanetary space; 3. to correlate the irradiation history of the grain to some of their bulk characteristics such as their carbon and methane contents. 


\section{The Solar Flare Record, Observed at Depth Greater than One Micron}

Observations were made with a scanning electron microscope on etched grain surfaces, after what we define as a 'strong' etching [8], enlarging the track diameter up to about $2000 \AA$. The track densities were measured in grains from the 100 to $400 \mathrm{Mesh}$ fractions extracted either from different samples of fines $(10084,12032,12070)$ or at different depths in core tube 12028. The track densities measured at the center of the grains ranged from $10^{7}$ to $10^{9}$ tracks $\mathrm{cm}^{-2}$ (Table I). No significant increase in these densities was observed when going from the 100 Mesh to the 400 Mesh grains, in the various samples. For a given grain, after the strong etching, the track densities measured on the external surface and at the center of a grain were about the same; this confirms that a high proportion $(\gtrsim 80 \%)$ of the grains do not show the homogeneous edge zoned track distribution - or track gradient - which can be expected for grains individually exposed in space to the very heavy (VH) nuclei of the solar flare cosmic rays and characterized by a drop in the track density by a factor of 2 to 3 on a depth of about $20 \mu$. Even the most favorable track gradient we found was not homogeneous

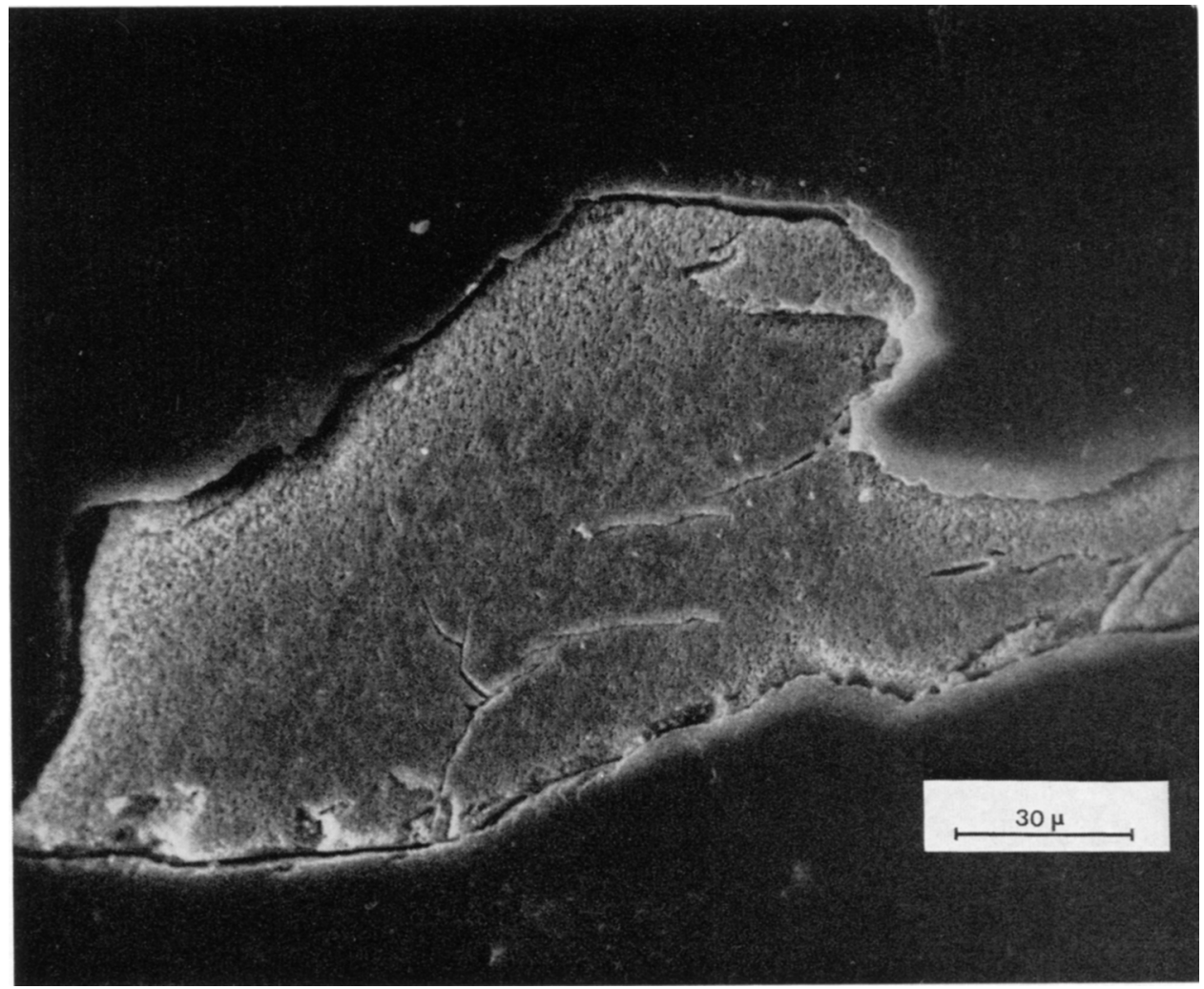

Fig. 1. 200 Mesh lunar pyroxene grain (sample 12028,55) showing a typical edge zoned track distribution. Less than $20 \%$ of the grains showed such a gradient generally considered as resulting from the 'individual' irradiation of the grains in solar flare cosmic rays. 


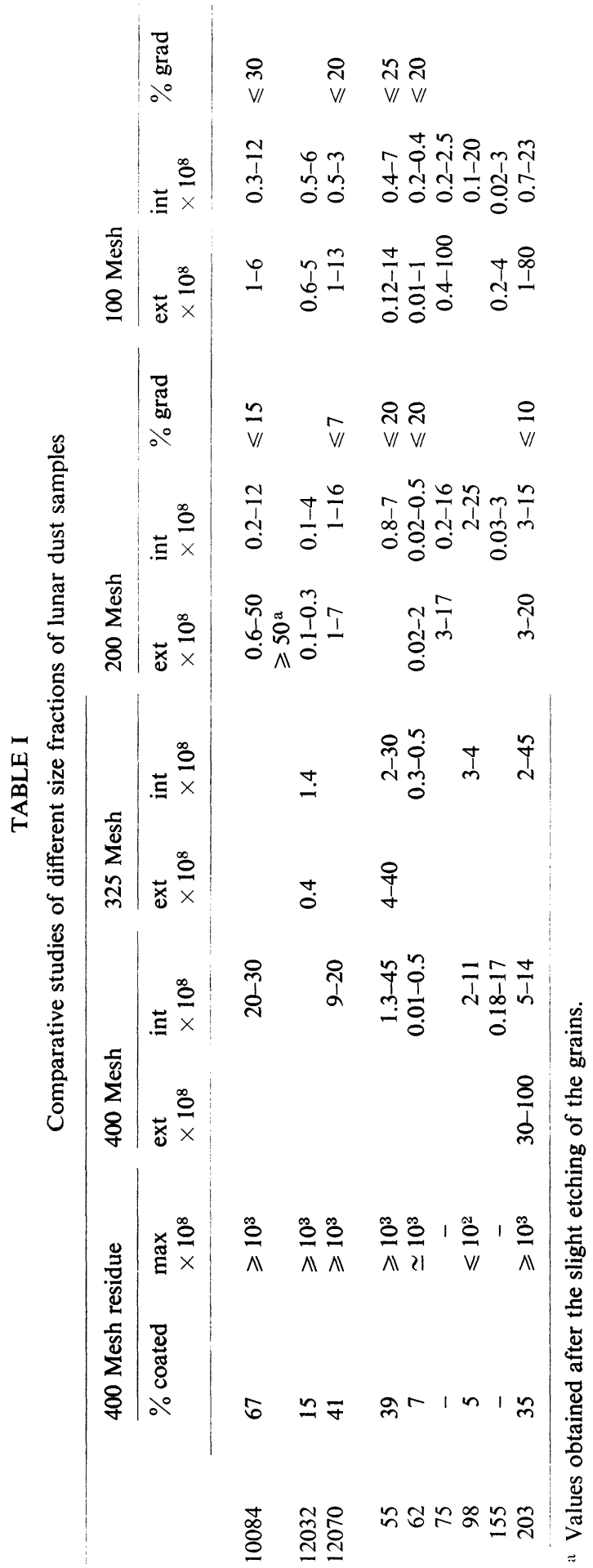




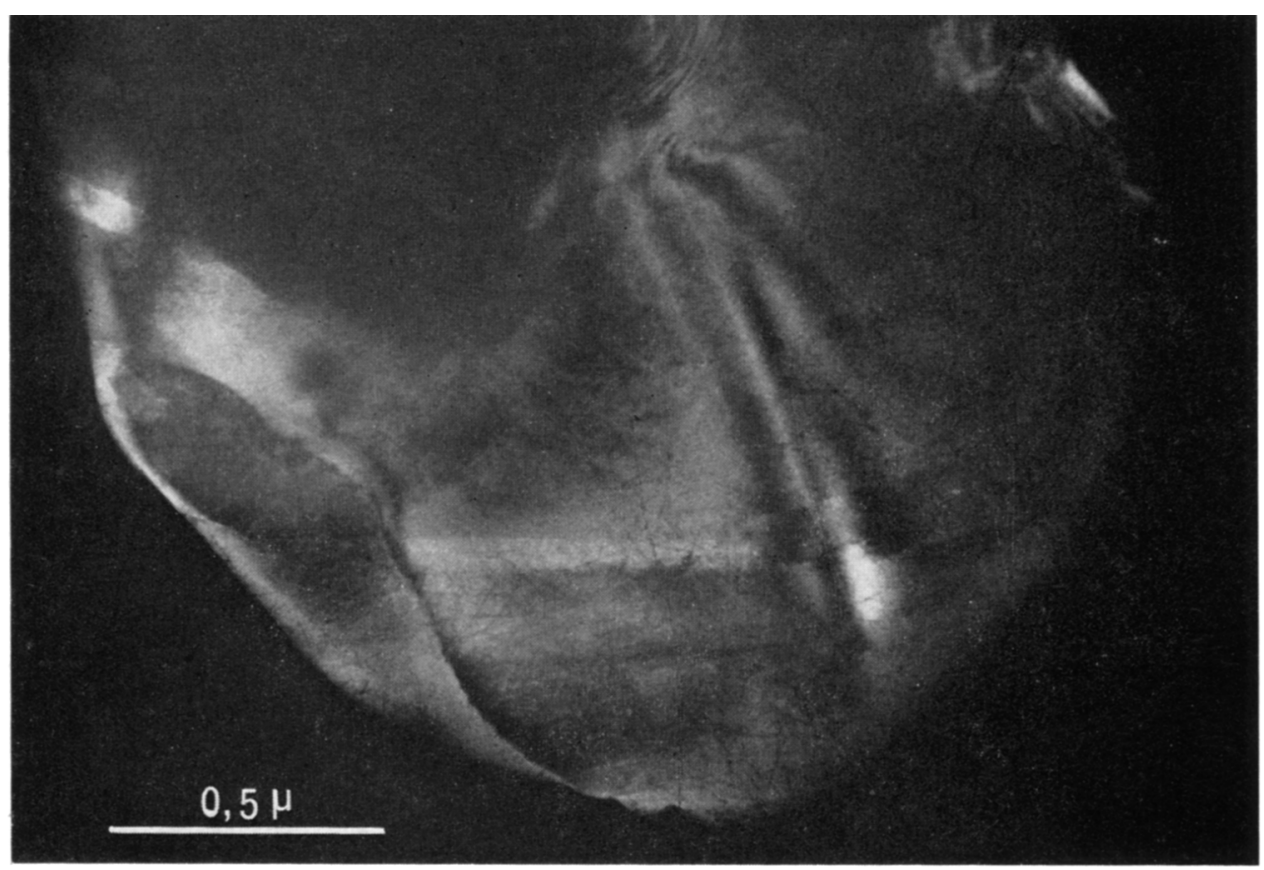

Fig. 2. $1 \mathrm{MeV}$ dark field micrograph of an Apollo 12 uncrushed dust grain containing a high density $\left(\sim 10^{11}\right.$ tracks $\left.\mathrm{cm}^{-2}\right)$ of latent nuclear particle tracks appearing as the lines of dark contrast.

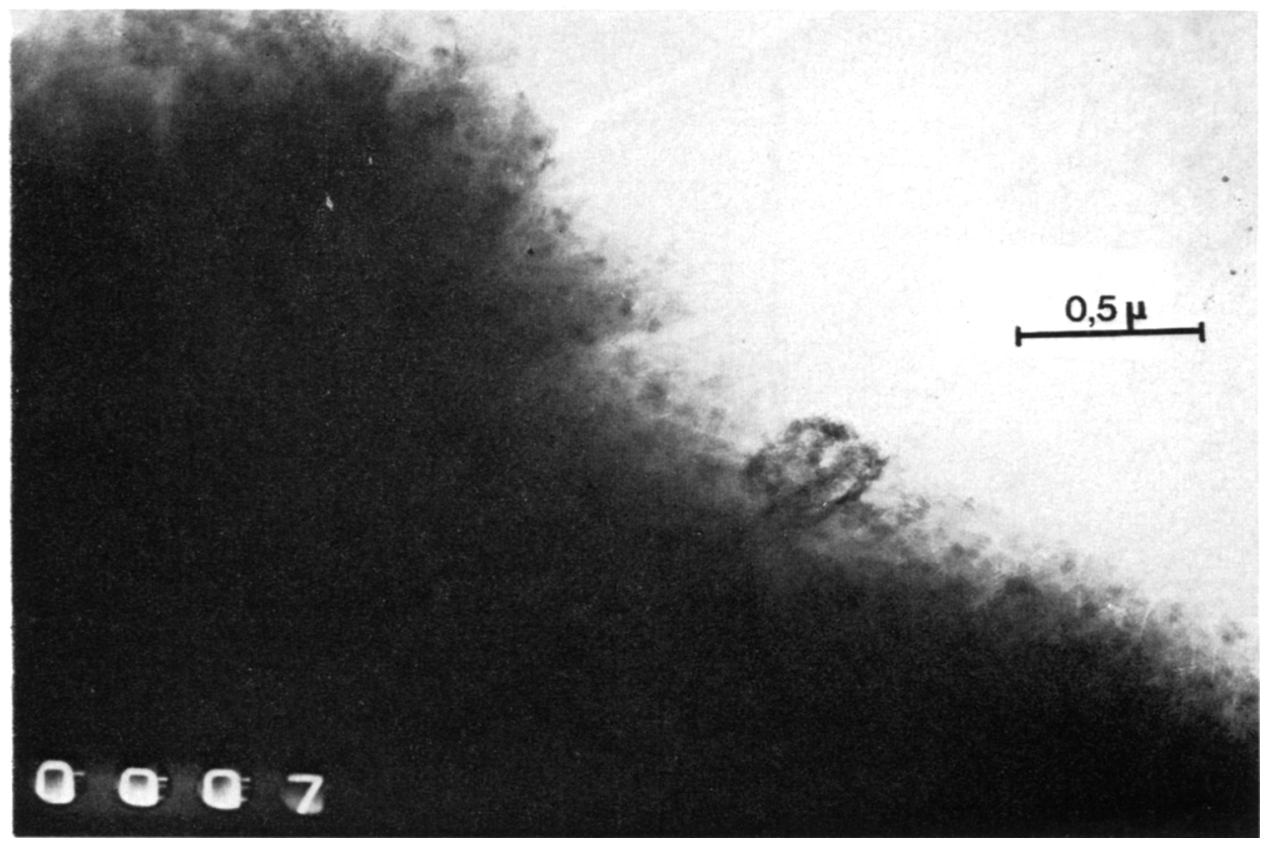

Fig. 3. $100 \mathrm{keV}$ micrograph of an Apollo 12 uncrushed dust grain, obtained thanks to the courtesy of the JEOL Company. This grain was 'slightly' etched and the tracks appear as the white shallow canals visible only on the edge of the grain. 


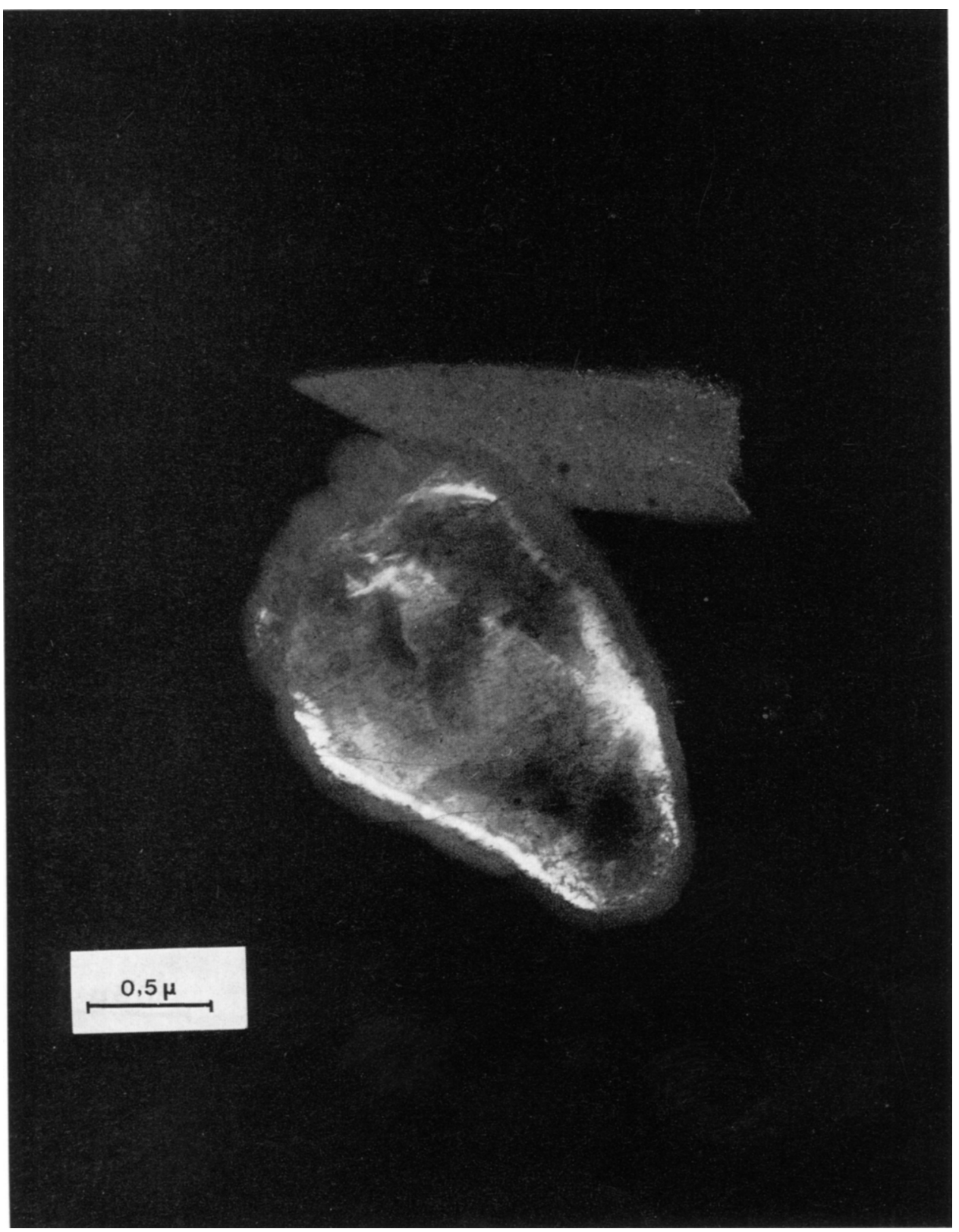

Fig. 4. $1 \mathrm{MeV}$ dark field micrograph of an Apollo 12 uncrushed dust grain, showing the superficial amorphous coating observed in crystals containing the highest track densities. 
(Figure 1) both along the edge of the grain and in its depth extension, and this does not facilitate the study of ancient solar flare VH nuclei, as discussed elsewhere [9].

No correlation of the track density with the depth was observed in core tube 12028 but a marked stratification was observed particularly distinct in sample 12028, 62.

Comstock et al. [10] and Crozaz et al. [11], who studied the track density distribution in the same core tube found only $20 \%$ of the grains showing a track gradient, as we did. On the contrary Arrhenius et al. [12] found measurable track gradients in a high proportion of the 100 Mesh grains showing the highest track densities $\left(\gtrsim 5.10^{8}\right.$ tracks $\mathrm{cm}^{-2}$ ). These authors find also an evident stratification in the core tube, and no correlation of the track density with the depth.

\section{The Ultramicroscopic Record in Uncrushed Micron Sized Dust Grains}

Before any etching the finest crystalline grains in the various dust samples generally contain high nuclear particle track densities which can exceed $10^{11}$ tracks $\mathrm{cm}^{-2}$ $[1,2,3,4,5,13)$ and which are only observable by high voltage $(1 \mathrm{MeV})$ transmission electron microscopy: they appear as lines of dark contrast in Figure 2. Furthermore, the grains are frequently rounded and covered with a superficial coating of amorphous material of about $500 \AA$ in thickness $[1,2,3]$ appearing as the dark lining surrounding the grain in Figure 3.

After a 'slight' chemical etching first developed by Barber et al. [13] the latent tracks are transformed in shallow etched canals with diameters of about $200 \AA$ (Figure 4) that C. Jouret has been able to observe currently with a $100 \mathrm{keV}$ electron microscope.

\section{The Ultramicroscopic Record in the Micron Sized Superficial Layer of the Coarser Grains}

We intended to verify if the high density of tracks observed in the finest grains were also registered in a micron-sized superficial layer in the coarser grains. Indeed during the 'strong' etching used for ordinary scanning electron microscope studies this layer could have been rubbed away thus preventing the observation of high track densities.

For this purpose, two types of experiments were performed: (1) five different size fractions of sample 12070 were crushed into micron sized fragments subsequently observed by transmission electron microscopies. We noted a marked increase in the probability of observing high latent and slightly etched track densities when going from the coarsest to the finest size fractions and this 'size effect' supports the hypothesis that the high track densities are registered in the most superficial layer in the grains; (2) external surfaces of $200 \mathrm{Mesh}$ feldspar and pyroxene grains from lunar sample 10084 were etched with the slight etching conditions used for transmission electron microscopy. Then we observed the external surface of the grains with a high 


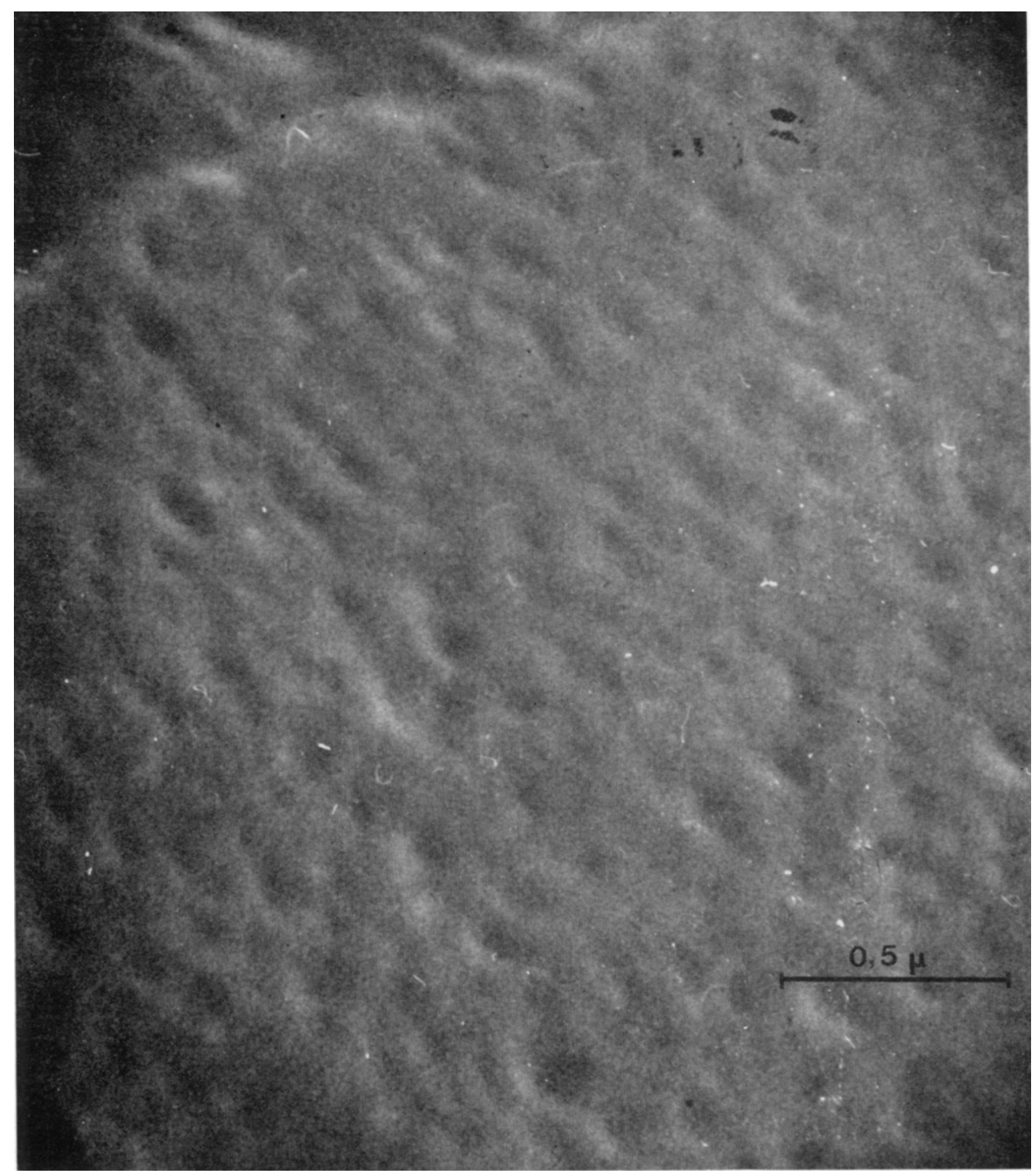

Fig. 5. High resolution scanning picture of the surface of a $200 \mathrm{Mesh}$ grain (sample 10084) which has been slightly etched. The track density is $\sim 2.10^{10}$ tracks $\mathrm{cm}^{-2}$.

resolution scanning electron microscope (JEOL) and detected high densities of tracks as those reported in Figure 5 and ranging from $5.10^{9}$ to $2.10^{10}$ tracks cm ${ }^{-2}$. Finally, some of these grains, either polished down or not, were then more strongly etched for 'ordinary' scanning electron microscopy and they definitively showed the lower track densities already noted by several groups for such etching conditions. In Figure 6, we reported our first preliminary attempts in plotting the variations of the track density with the depth in the most superficial micron-sized layer of the grains. 


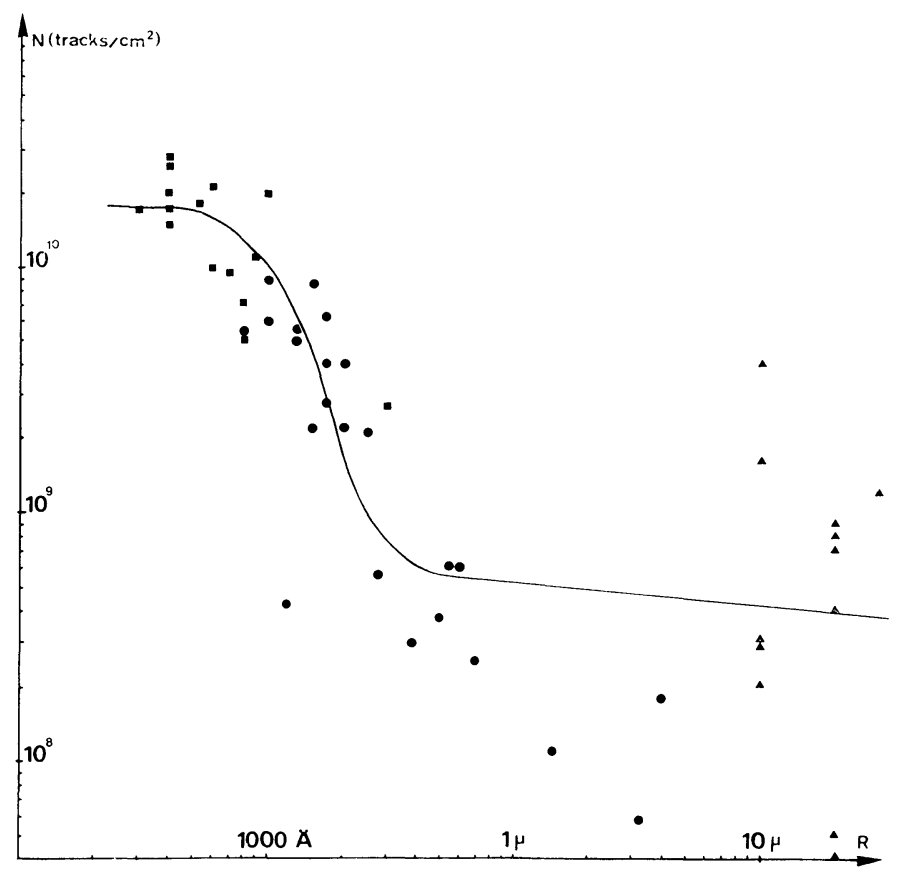

Fig. 6. Track density as a function of the depth $R$ in 200 Mesh grains of sample 10084. The squares and the dots represent the values measured on the external surfaces of the grains after a 'slight' and a 'strong' etching respectively. For these points we plotted the track density as a function of the track diameter which gives a rough estimate for $R$. The triangles figure the values obtained for another set of grains on internal polished surfaces, at depth $R$ directly measured on the SEM micrographs.

\section{Dynamic Processes at the Surface of the Moon}

Arrhenius et al. [12], Comstock et al. [10] and Crozaz et al. [11] have suggested that the solar flare irradiation of the dust grains most likely started when they were released in the lunar soil. In a given layer the variations observed between the track densities are then explained by differences in residence times at the top of the regolith, with the samples showing a gradient having been the closest to the surface.

At the present time we slightly favor another possible irradiation history for the grains suggested to us by M. Maurette and occurring in two successive steps:

(1) first, the grains were mostly loaded with tracks at depths further than $1 \mu$ when they were part of the top surface of a rock. Then, the variations in track densities in the lunar dust grains could be explained by the variations in the depths at which they got 'excavated' from the rocks most likely by micrometeorite impacts. This conclusion is supported by the greater proportion of glassy spherules showing a track gradient [14] and by roughly the similar track density distribution (ranging from $10^{7}$ to $10^{9}$ tracks $\mathrm{cm}^{-2}$ ) we obtained by crushing a mm sized fragment artificially 'excavated' from the top surface of lunar rock 10047. This interpretation also explains why the $400 \mathrm{Mesh}$ grains are not more loaded with 'volume' tracks than the $100 \mathrm{Mesh}$ grains, but it 
cannot account easily for the marked decrease in the track densities in sample12028,62.

(2) then, the grains spend a too short time $-10^{3}$ to $10^{4}$ yr only - in the most superficial layers of the regolith to get a solar flare track contribution exceeding that acquired before their release in the lunar soil, thus explaining the 'lack' of track gradient. But during this short time, they were bombarded superficially with solar wind nuclei responsible for the amorphous coating on the grain $[1,2,3,4,5]$ and with heavy solar ions with energies intermediate between those of the solar wind nuclei and those of the more energetic solar flare cosmic rays producing high track densities in the superficial micron sized layer in the grains.

The simultaneous implantation of solar wind and low energy solar ions is evidenced by the correlation between the high track densities and the existence of an amorphous coating in the smallest grains (Table I) and argues against a cosmic dust origin for the finest fraction as suggested by Barber et al. [13] because it is very difficult to explain that such a regular coating could have survived an impact with the Moon. To check further the validity of this irradiation scheme for the dust grains, it will be interesting to apply the methods described in the next chapter to verify if there is a correlation between the high track density measured in the most superficial layer of the 200 Mesh grains and those measured at depths greater than one micron in the same grains.

\section{Evidences for Suprathermal Ions in the Interplanetary Space}

Barber et al. [13] favor the hypothesis that the micron sized dust grains are cosmic dust particles accreted by the Moon and loaded in the interplanetary space by solar flare cosmic rays tracks. They regard it as "highly unlikely that suprathermal heavy ions were responsible of these high track densities" - as we much earlier suggested $[4,5]$ - because: (1) the energy of suprathermal ions is below the sensibility threshold for track registration; (2) the high track densities are registered in the volume of the grains and therefore no steep surface track gradient can be observed.

We want to make the following comments concerning these criticisms:

(1) The curve reported in Figure 6 shows that a steep track gradient is indeed observed in the most superficial micron-sized layer of the grains. By supposing that the tracks are registered on the whole range of the ions, the curve in Figure 6 represents a rough integrated range spectrum for the particles : $N\left(R>R_{0}\right)=f\left(R_{0}\right)$, where $N$ represents the tracks with range $R$ greater than $R_{0}$ and from which we can deduce a differential energy spectrum $\mathrm{d} N / \mathrm{d} E=f(E)$ by computing the quantity $(\mathrm{d} N / \mathrm{d} R) \times$ $\times(\mathrm{d} R / \mathrm{d} E)$. This differential spectrum is shaped like a pulse. Therefore it looks similar to that measured for suprathermal protons by Franck [6] but is very different from the power law spectrum which can be extrapolated from the most recent data of Armstrong et al. [15] and Stone [16], for solar flare VH nuclei with energy $\gtrsim 1 \mathrm{MeV} / \mathrm{amu}$.

(2) In a paper which will be published elsewhere [17] we will show that nickel ions with energies down to $0.02 \mathrm{MeV} /$ nucleon can produce tracks in silicate grains and therefore the most severe objection to the existence of heavy suprathermal ions is no longer valid. 


\section{Correlation with Other Results}

The Orsay group has already discussed some correlations between the microscopic irradiation features in the grains and the bulk mechanical and optical properties of the regolith $[4,5]$. We will mention here a new development in these correlation studies.

Cadogan et al. [18] have shown that part of the indigenous methane and ethane contents in the lunar fines seems to be correlated to the solar flare irradiation of the grains exposed unshielded on the lunar surface as described in the work of Arrhenius et al. [12] and to the fraction of grains with an amorphous coating as suggested by us. These results seem to indicate that part of the indigeneous methane in the lunar dust could have been synthesized by an ancient solar wind implantation in the grains. The Orsay group is currently verifying these important conclusions in analyzing the irradiation record in dust samples simultaneously studied by the Bristol group and in conducting artificial solar wind type implantations in micron-sized fragments obtained by crushing internal chunk of various types of lunar rocks.

\section{Acknowledgements}

All the transmission microscope observations were made by L. Durrieu and C. Jouret at the Institut d'Optique Electronique du CNRS, Toulouse, France. We acknowledge the generous help of the JEOL Company for the scanning electron microscope observations. We are indebted to Dr R. Bernas for his very active support and interest. We wish to express our gratitude to Dr M. Maurette for many stimulating discussions.

\section{References}

[1] Borg, J., Dran, J. C., Durrieu, L., Jouret, C., and Maurette, M.: 1970, Earth Planetary Sci. Letters 8, 379.

[2] Dran, J. C., Durrieu, L., Jouret, C., and Maurette, M.: 1970, Earth Planetary Sci. Letters 9, 391.

[3] Borg, J., Dran, J. C., Durrieu, L., Jouret, C., and Maurette, M.: 1970, 'High Voltage Electron Microscope Studies of Extraterrestrial Matter', 7th Int. Colloquium Corpuscular Photography and Visual Solid Detectors, Barcelona, Paper No. 41.

[4] Borg, J., Durrieu, L., Jouret, C., and Maurette, M.: 1971, 'The Ultramicroscopic Irradiation Record of Micron-Sized Lunar Dust Grains', Second Lunar Science Conference (unpublished proceedings).

[5] Borg, J., Durrieu, L., Jouret, C., and Maurette, M.: 1971, Geochim. Cosmochim. Acta (in press).

[6] We give only the references of the most recent contributions actually in press in Geochim. Cosmochim. Acta (October 1971): Arrhenius, G., Liang, S., MacDougall, D., Wilkening, L., Bhandari, N., Bhatt, S., Lal, D., Rajagopalan, G., Tamhane, A. S., and Venkatavaradan, V. S.: 'The Exposure History of the Apollo 12 Regolith'.

Barber, D. G., Cowsik, R., Hutcheon, I. D., Price, P. B., and Rajan, R. S.: 'Solar Flares, the Lunar Surface and Gas-Rich Meteorites'.

Borg, J., Durrieu, L., Jouret, C., and Maurette, M.: 'Ultramicroscopic Features in MicronSized Lunar Dust Grains and Cosmophysics'.

Crozaz, G., Walker, R. M., and Woolum, D.: 'Nuclear Track Studies of Dynamic Surface Processes on the Moon and the Constancy of Solar Activity'.

Fleischer, R. L., Hart, H. R., and Comstock, G. M.: 'Very Heavy Solar Cosmic Rays: Energy Spectrum and Implication for Lunar Erosion'. 
[7] Frank, L. A.: 1970, J. Geophys. Res. 75, 707.

[8] Bastin, G., Borg, J., Dran, J. C., Maurette, M., and Vassent, B.: 1970, 'Etude de l'enregistrement de traces d'interactions nucléaires dans des pyroxènes et des feldspaths d'origine lunaire, météoritique et terrestre', Proceedings of the VIIth International Colloquium on Corpuscular Photography and Visual Solid Detectors, Barcelona, July 1970.

[9] Dran, J. C., Duraud, J. P., and Maurette, M.: 1972, this volume, p. 309.

[10] Comstock, G. M., Evwaraye, A. O., Fleischer, R. L., and Hart, H. R. Jr.: 1971, 'The Particle Track Record of Lunar Soil', General Electric Company, Report No. 71-C-073.

[11] See for example the work of Crozaz et al. in [6].

[12] See for example the work of Arrhenius et al. in [6].

[13] Barber, D. J., Hutcheon, I., and Price, P. B.: 1971, Science 171, 372.

[14] Crozaz, G., Haack, U., Hair, M., Maurette, M., Walker, R. M., and Woolum, D.: 1970, Geochim. Cosmochim. Acta Suppl. 1 3, 2051.

[15] Armstrong, T. P. and Krimigis, S. M.: 1970, 'A Statistical Study of Solar Protons, Alphas and $Z>3$ Nuclei in 1967-68', Johns Hopkins Univ. Preprint.

[16] Stone, E.: 1971, 'Low Energy Solar Cosmic Rays', Caltech Preprint.

[17] Borg, J., Maurette, M., and Vassent, B.: 1971, 'Low Energy Solar Nuclear Particles: New Detection Methods and New Results', Paper to be presented at the 12th Int. Conf. on Cosmic Rays. Hobart, Australia (August 1971).

[18] Cadogan, P. H., Eglinton, G., Maxwell, J. R., and Pillinger, C. T.: 1971, Nature 231, 29. 